\title{
BMJ Open Factors associated with HPV and other self-reported STI coinfections among sexually active Brazilian young adults: cross-sectional nationwide study
}

\author{
Natalia Luiza Kops, ${ }^{1,2}$ Marina Bessel, ${ }^{1}$ Jaqueline Driemeyer Correia Horvath, ${ }^{1}$ \\ Carla Domingues, ${ }^{3}$ Flávia Moreno Alves de Souza, ${ }^{4}$ Adele Schwartz Benzaken, ${ }^{4}$ \\ Gerson Fernando Mendes Pereira, ${ }^{4}$ Ana Goretti Kalume Maranhão, ${ }^{3}$ \\ Luisa Lina Villa, ${ }^{5}$ Barbara Mello, ${ }^{6}$ Eliana Marcia Wendland ${ }^{1,7}$
}

To cite: Kops NL, Bessel M, Horvath JDC, et al. Factors associated with HPV and other self-reported STI coinfections among sexually active Brazilian young adults: cross-sectional nationwide study. BMJ Open 2019;9:e027438. doi:10.1136/ bmjopen-2018-027438

- Prepublication history and additional material for this paper are available online. To view these files, please visit the journal online (http://dx.doi. org/10.1136/bmjopen-2018027438).

Received 22 0ctober 2018 Revised 19 February 2019 Accepted 4 April 2019

\section{ABSTRACT}

Objectives To analyse factors associated with genital human papillomavirus (HPV) and other self-reported sexually transmitted infection (STI) coinfections among women and men aged 16-25 years from Brazil.

Design A cross-sectional, nationwide, multicentre study. Setting 119 primary healthcare centres between September 2016 and November 2017.

Participants 6388 sexually active young adults were enrolled by trained health professionals.

Primary outcome measure Genital HPV and other selfreported STI coinfections.

Results 0 3512 participants with valid data for genital HPV and (STI)-positive status, 276 (9.60\%, 95\% Cl 7.82\% to $11.36 \%$ ) had HPV/STI coinfection. Among men, HPV/ STI coinfection was more prevalent than HPV infection alone. Among HPV-positive participants, the percentage of subjects who reported having another STI was highest for gonorrhoea at $4.24 \%(95 \% \mathrm{Cl} 2.67 \%$ to $5.81 \%)$, followed by syphilis, herpes and HIV. Smoking, drug use and ever having a same-sex sexual experience were risk factors that were uniquely associated with HPV/STI coinfection compared with HPV infection alone.

Conclusions The results identified a low prevalence of self-reported STIs, but in participants with at least one STI, the prevalence of HPV was high. These results reinforce the importance of implementing strategies to prevent risky behaviours among Brazilian young adults.

\section{INTRODUCTION}

Sexually transmitted infections (STIs) are one of the most common global health problems; more than 1 million STIs are estimated to be acquired daily worldwide. ${ }^{1}$ Human papillomavirus (HPV) infection is one of the most prevalent STIs, affecting nearly half of the population in some age groups in countries such as the USA and Brazil. ${ }^{2-4}$

HPV is a group of more than 150 related viruses, and at least 13 types are considered oncogenic, presenting a higher risk or
Strengths and limitations of this study

- Risk factors associated with human papillomavirus and other self-reported sexually transmitted infection (STI) coinfections were analysed among sexually active young adults who used the public health system.

- This study involved a nationwide sample that represented a large and diverse population.

- STI cases were self-reported, and many STIs have no signs or symptoms, both of which may have contributed to an underestimation of STI prevalence.

probability of persistent infections and being associated with precursor cancer lesions. ${ }^{5}$ Although most HPV infections do not cause symptoms, persistent HPV infection can cause cervical cancer in women. This cancer is the fourth-most common cancer among women, and $99 \%$ of cases are linked to genital infection with HPV. ${ }^{6}$ Furthermore, there is growing evidence that HPV is a key factor in the development of other anogenital cancers (anal, vulvar, vaginal and penile cancers) as well as head and neck cancers. ${ }^{7}$

Since high-risk strains of HPV is a necessary but not a sufficient cause of cervical cancer other biological, behavioural and environmental cofactors have been implicated in the development of HPV-associated cancers. ${ }^{8}$ Studies establishing an association between HPV infection and other STIs may help to improve understanding of the role of these coinfections in the development of cervical cancer. Interactions among HPV, other viruses and microbes that share a similar epithelial niche could enhance HPV replication and infection persistence and accelerate cancer progression. 
Precise estimates of STI coinfection are limited, especially in the general population. ${ }^{1}$ The availability of epidemiological data on HPV and STI coinfection in Brazilian young adults is essential for the development of successful strategies for STI prevention, diagnosis and management in the country. According to this epidemiological scenario, the present study aimed to analyse factors associated with genital HPV and other self-reported STI coinfections among women and men aged 16-25 years from Brazil.

\section{METHODS}

This is a nationwide, cross-sectional, multicentre study of sexually active young adults who use the public health system (POP-Brazil Study). ${ }^{9}$ Participants from 119 designated public primary healthcare units of the 26 state capitals and the Federal District of Brazil were invited to participate from September 2016 to November 2017. Primary healthcare professionals were personally trained by the central study coordinator for data acquisition.

The following exclusion criteria were applied: pregnant women, those who delivered a baby in the last 3 months, who had undergone a hysterectomy or trachelectomy and who had any history of cervical intraepithelial neoplasia grade 2 or higher. Participants vaccinated for HPV were excluded from the analyses.

The study was approved by the Ethics Committee on Human Research (Hospital Moinhos de Vento-protocol no. 1607032) and by the recruitment sites. All participants provided written consent.

\section{Patient and public involvement}

Patients and the public were not involved in the protocol development.

\section{Study procedures}

All individuals answered a standardised questionnaire based on validated instruments ${ }^{10}$ that assessed sociodemographic characteristics, ${ }^{11}$ sexual behaviours, smoking status $^{12}$ and history of STIs. ${ }^{13}$ Participants were asked about their income, education level, age at first intercourse, age at first pregnancy, age of partner at first sexual intercourse, number of sexual partners in the last year, whether they had ever had a same-sex sexual experience, current condom use and condom use at first sexual intercourse. To evaluate alcohol and drug consumption, we asked about patterns of use throughout life, use in recent years and current use.

Cervical samples were obtained using a digene HC2 DNA Collection Device (Qiagen), and penile samples (penile shaft, glans penis/coronal sulcus, scrotum) were obtained using a Dacron swab (Qiagen) previously humidified in sterile saline solution. ${ }^{9}$ All biological samples were maintained at room temperature $\left(15^{\circ} \mathrm{C}\right.$ to $\left.25^{\circ} \mathrm{C}\right)$ according to the manufacturer's instructions and transported to a central laboratory for specimen processing.
HPV detection and typing were performed using the Linear Array HPV Genotyping Test (Roche Diagnostics), which is based on PCR amplification (fragment of 450 base pairs of the polymorphic region of the L1 gene of HPV), followed by specific hybridisation and colourimetric detection, in a certified laboratory. The amplification of human $\beta$-globin (biotinylated primers $\mathrm{GH} 2 \mathrm{O}$ and PC04, 268 base-pair fragments) was used as an internal control.

To characterise other STIs, we asked participants if they had ever been diagnosed with syphilis, gonorrhoea and/or herpes. We also asked if they had ever been tested for HIV and for the results of any previous tests. Additionally, participants were invited to undergo a rapid HIV test. Individuals who reported having STIs had a positive HIV test result in the past or had a positive HIV rapid test result at the time of the interview were considered positive.

Unanswered questions were considered missing values in the analysis. Those who were never tested for HIV and who did not take a rapid or laboratorial test at the time of the interview were also considered to have missing values for HIV.

Participants were categorised into three groups according to their infection status: HPV only, HPV/STI coinfection or STI only. HPV only was defined as having just HPV without other STIs; HPV/STI coinfection was defined as having any one type of HPV plus any one of the four STIs and STI only was defined as having one or more of the four STIs without HPV.

The sample size was based on the main research project aim, namely, establishing the prevalence of HPV in Brazil. The number of samples were equally divided in all regions to maximise diversity in less-populated areas.

\section{Statistical analysis}

Categorical variables were summarised using absolute frequencies and percentages, while continuous variables were analysed using means and CIs. To compare proportions, a $\chi^{2}$ test was used. For differences among the three infection groups, Poisson regression models with robust variance and multiple comparison Tukey tests were used. For the evaluation of risk factors associated with HPV/STI coinfection, a Poisson model with robust variance was estimated to compare those with HPV/STI coinfection to those infected by HPV only. All variables with $\mathrm{p}<0.20$ were included hierarchically (sociodemographic and socioeconomic, behavioural and sexual characteristics, consecutively) in a multivariate model. The interactions among effect modifiers were analysed.

To adjust the distribution of the sample to the study population, we weighted the measures by the size of the population in each capital and by sex. Therefore, all results are reported as weighted. Statistical analysis was performed using SAS software V.9.4 (Statistical Analysis System, SAS Institute), and statistical significance was defined as $\mathrm{p}<0.05$. 


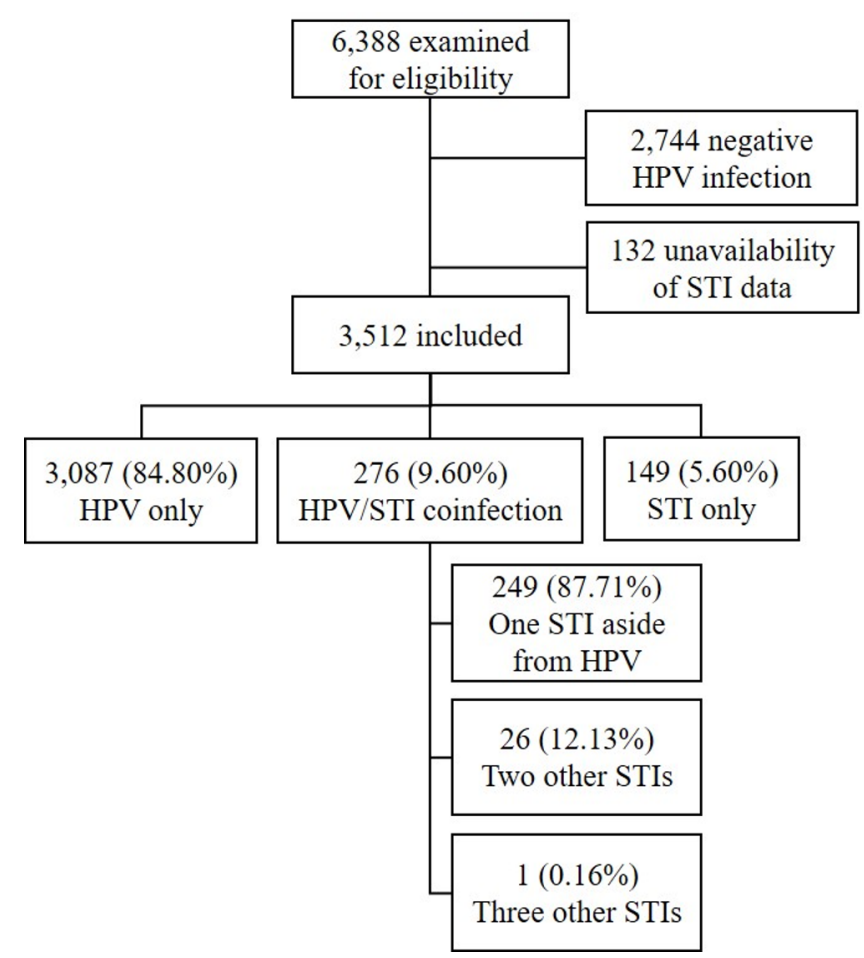

Figure 1 Fluxogram of the participants included in the study. HPV, human papillomavirus; STI, sexually transmitted infection.

\section{RESULTS}

In total, 6388 participants (5268 women) were included in the study (figure 1). The predominant socioeconomic index was class C (55.46\%, 95\% CI 53.30 to 57.62), and the predominant skin colour was pardo/brown $(56.97 \%$, $95 \%$ CI 54.77 to 59.16$)$. The analysis was restricted to HPV-positive participants and those that provided information on STI status (see online supplementary file 1). Among the 3512 participants, 276 (9.60\%, 95\% CI 7.82 to 11.36) had HPV/STI coinfection. Of these individuals, the majority $(87.71 \%)$ reported having only one STI aside from HPV.

Among women, HPV infection only was more prevalent than HPV/STI coinfection. In contrast, HPV/STI coinfection was more prevalent than HPV infection only among men. Participants who reported having other STIs only were older than those who reported having HPV infection only, but no age difference was found in relation to HPV/STI coinfection. Furthermore, sociodemographic and socioeconomic characteristics, such as skin colour, income and education level were similar between the two groups. Participants with HPV/STI coinfection were younger at first sexual intercourse than those with HPV only ( 14.90 years, $95 \%$ CI 14.46 to 15.34 vs 15.29 years, $95 \%$ CI 15.16 to 15.43$)$. On the other hand, the age of the partner at first sexual intercourse was higher in the HPV/ STI coinfection group than in the other groups (table 1).

Among the HPV-positive participants, gonorrhoea was the most prevalent reported infection $4.24 \%$ (95\% CI $2.67 \%$ to $5.81 \%$ ), followed by syphilis, herpes and HIV (figure 2). When we look for the prevalence of HPV among all participants who reported STI, people with HIV presented the higher HPV positivity, with 25 positive cases of HPV among $33 \mathrm{HIV}$ cases $(85.41 \%$, 95\% CI $69.89 \%$ to $100.00 \%)$. The second STI most associated with HPV was gonorrhoea: 76 of 133 cases $(62.77 \%, 95 \%$ CI $46.66 \%$ to $78.88 \%)$, followed by herpes $(62.23 \%, 95 \%$ CI $46.55 \%$ to $77.92 \%)$ and syphilis $(59.56 \%, 95 \%$ CI $49.07 \%$ to $70.05 \%)$ (data not shown).

The prevalence of HPV/STI coinfection varied by sex. More men had HPV/gonorrhoea and HPV/HIV coinfection than women (figure 3). Regarding STI treatment, $85.97 \%$ (95\% CI $82.43 \%$ to $89.52 \%$ ) of participants searched for health professionals, $7.49 \%$ (95\% CI $4.59 \%$ to $10.39 \%$ ) did nothing, and $6.53 \%$ (95\% CI $4.28 \%$ to $8.77 \%)$ were self-medicated; no significant sex differences emerged $(\mathrm{p}=0.632)$ (data not shown).

A multivariate analysis was performed to verify the variables associated with HPV/STI coinfection. When adjusted for sociodemographic and socioeconomic variables, the male sex was associated with coinfection. However, after the inclusion of behavioural and sexual variables, sex was no longer significant. In the full model (model 3), smoking (prevalence ratio $(\mathrm{PR})=1.64$, 95\% CI 1.03to 2.61), illegal drug use ( $\mathrm{PR}=1.58,95 \% \mathrm{CI}$ 1.05 to 2.39 ) and same-sex sexual experience ( $\mathrm{PR}=2.15$, $95 \%$ CI 1.27 to 3.62 ) were variables that were uniquely associated with coinfection compared with those associated with HPV infection only (table 2). However, the estimated indirect effect mediated by gender was 0.007 $(p=0.004)$, and $10.55 \%$ of the total effect of same-sex sexual experience was mediated by sex. Education and socioeconomic class were not associated with the presence of coinfection.

\section{DISCUSSION}

In this study, the prevalence of HPV/STI coinfection among 3512 Brazilian young adults was analysed. This study included a nationwide sample that represented a large and diverse population and showed important data on risk factors associated with HPV/STI coinfection. $\mathrm{HPV} /$ gonorrhoea was the most prevalent coinfection, followed by HPV/syphilis. Smoking, drug use and ever having a same-sex sexual experience were risk factors that were uniquely associated with HPV/STI coinfection compared with HPV infection only.

There is limited information about HPV/STI coinfection at the population level in Brazil. Although several studies have evaluated coinfection among STIs, few studies have evaluated HPV/STI coinfection. When comparing studies of the prevalence of HPV coinfection, care should be taken regarding the types of STIs included and the site of research. For example, an African study found an HPV coinfection prevalence of $44.0 \%,{ }^{14}$ similar to other African study witch found $47 \%$, but they also included chlamydia infection. ${ }^{15}$ Furthermore, the majority of studies have been performed in small female samples ${ }^{16}$ 
Table 1 Characteristics of the participants according to the presence of HPV infection and other self-reported STIs in the POP-Brazil Study, 2016-2017

\begin{tabular}{|c|c|c|c|c|}
\hline Variables & $\begin{array}{l}\text { Total } \\
3512 \\
\end{array}$ & $\begin{array}{l}\text { HPV only } \\
3087(84.80)\end{array}$ & $\begin{array}{l}\text { HPV/STI coinfection } \\
276(9.60)\end{array}$ & $\begin{array}{l}\text { STI only } \\
149(5.60)\end{array}$ \\
\hline \multicolumn{5}{|l|}{ Sex } \\
\hline Male & 621 & $500(33.73)^{\star} \dagger$ & $80(47.20)^{*}$ & $41(54.20) \dagger$ \\
\hline \multicolumn{5}{|l|}{ Age (years) } \\
\hline \multicolumn{5}{|l|}{ Race/skin colour } \\
\hline White & 808 & $696(21.66)$ & $74(24.07)$ & $38(20.44)$ \\
\hline Black & 569 & $496(17.07)$ & $44(17.18)$ & $29(20.72)$ \\
\hline Pardo/brown & 2038 & $1808(59.26)$ & $149(56.03)$ & $81(57.86)$ \\
\hline \multicolumn{5}{|l|}{ Relationship status } \\
\hline Without a partner & 884 & $763(24.89)$ & $72(30.58)$ & $49(30.76)$ \\
\hline Current condom use & 1773 & $1583(51.76) \dagger$ & $128(46.35)$ & $62(33.69) \dagger$ \\
\hline Condom use at first sexual intercourse & 2246 & $2001(60.07)$ & $161(59.05)$ & $84(54.57)$ \\
\hline Ever been pregnant & 1294 & $1114(43.94) \dagger$ & $114(51.44)$ & $66(63.87) \dagger$ \\
\hline Ever had same-sex sexual experience & 158 & $122(5.25)^{\star} \dagger$ & $20(18.29)^{\star}$ & $16(24.41) \dagger$ \\
\hline Alcohol consumption & 2592 & $2250(73.56)^{\star}$ & $231(86.04)^{*}$ & $111(75.53)$ \\
\hline Drug use & 1009 & $823(29.79)^{\star}$ & $133(56.16)^{\star} \ddagger$ & 53 (33.88)‡ \\
\hline \multicolumn{5}{|l|}{ Smoking status } \\
\hline Non-smoker & 2326 & $2099(64.74)^{\star}$ & $139(42.09)^{\star} \ddagger$ & 88 (63.21)‡ \\
\hline \multicolumn{5}{|l|}{$\begin{array}{l}\text { Number of sexual partners in the last } \\
\text { year }\end{array}$} \\
\hline$\geq 2$ & 227 & $952(34.14)^{\star} \dagger$ & $129(53.20)^{*}$ & $56(53.04) \dagger$ \\
\hline$<2$ & 1137 & $2010(65.86)^{\star} \dagger$ & $128(46.80)^{\star}$ & 89 (46.96)† \\
\hline
\end{tabular}

*Significant differences between HPV only and HPV/STI coinfection groups $(P<0.05)$.

$\dagger$ Significant differences between HPV only and STI only groups $(P<0.05)$.

‡ Significant differences between STI only and HPV/STI coinfection groups $(\mathrm{P}<0.05)$.

Results of Poisson regression analysis and Tukey's post-hoc multiple comparison tests.

HPV, human papillomavirus; STI, sexually transmitted infection.

and in target populations, such as those who are pregnant, ${ }^{17}$ homosexual ${ }^{18}$ or HIV positive. ${ }^{7}$

Despite the low frequency of self-reported STIs during the short sexual life of the study participants, the prevalence of genital HPV was high among participants with STIs. Genital infections with HPV are dynamic, usually occurring soon after acquisition of a new sexual partner and regressing after immune recognition, although occasionally persisting for years. Furthermore, with the presence of any STI, HPV infection is even more prevalent, and a history of prior STIs has been linked to a reduced ability to clear HPV or an increased risk of acquisition of HPV infection. ${ }^{19}$

Among HPV-infected women, concurrent infections with chlamydia, gonorrhoea and herpes have been linked to the persistence of HPV and an increased risk of cervical neoplasia. ${ }^{20}$ HIV predisposes affected women to acquire HPV infections and also promotes the development of cervical neoplasia through the upregulation of HPV oncogene expression. ${ }^{21}$ The prevalence of HPV in HIV-positive 


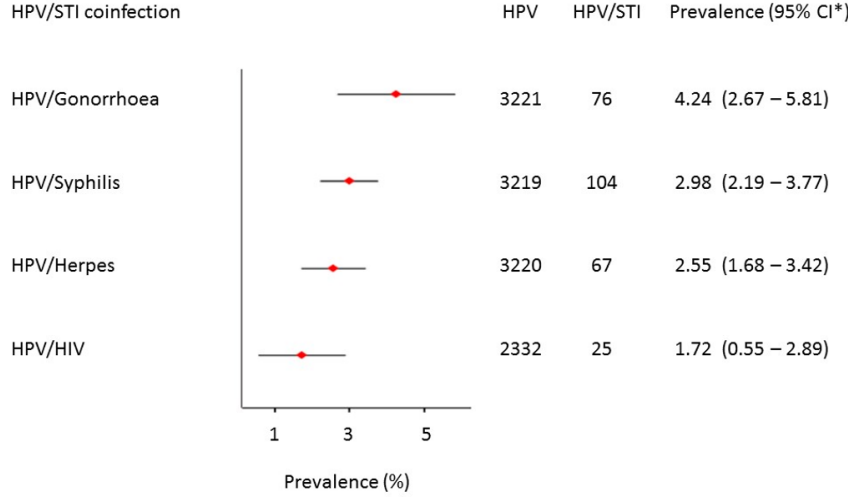

Figure 2 Prevalence of STIs among the HPV-positive participants. *Weighted by sex and size of population in each capital. The lines indicate $95 \%$ Cls. HPV, human papillomavirus; STI, sexually trasmitted infection.

young adults $(85.4 \%)$ was higher than that in Puerto Rico $(50.3 \%)^{22}$ and Italy $(48 \%)^{23}$ but similar to that in other previous studies in Brazil $\left(68.1 \%^{24}\right.$ and $73.5 \%{ }^{25}$ ) and sub-Saharan Africa (72.2\%) ${ }^{26}$ In contrast, the low rate of HPV/HIV coinfection could be explained by the age of

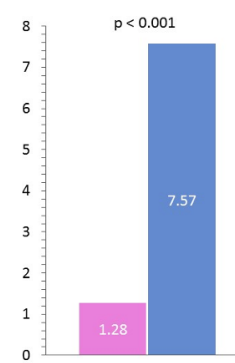

Gonorrhea/HPV

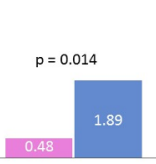

HIV/HPV

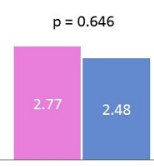

Syphilis/HPV

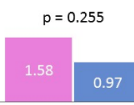

Herpes/HPV m Female $=$ Male

Figure 3 Prevalence of HPV and other sexually transmitted infections between sex. HPV,human papillomavirus.

the study population because HPV is acquired via sexual exposures; newly sexually active adolescents and young adults have the highest risk of HPV acquisition, ${ }^{27}$ while young adults have not had sufficient time to develop other STIs. The same phenomenon occurs with HPV/ herpes coinfection, which was also not as common in this study as in other studies, in addition to robust epidemiological evidence suggesting that HPV could be a possible cofactor in cervical carcinogenesis. ${ }^{28}$

Table 2 Prevalence ratios of associated factors and HPV/STI coinfection. POP-Brazil Study, 2016-2017

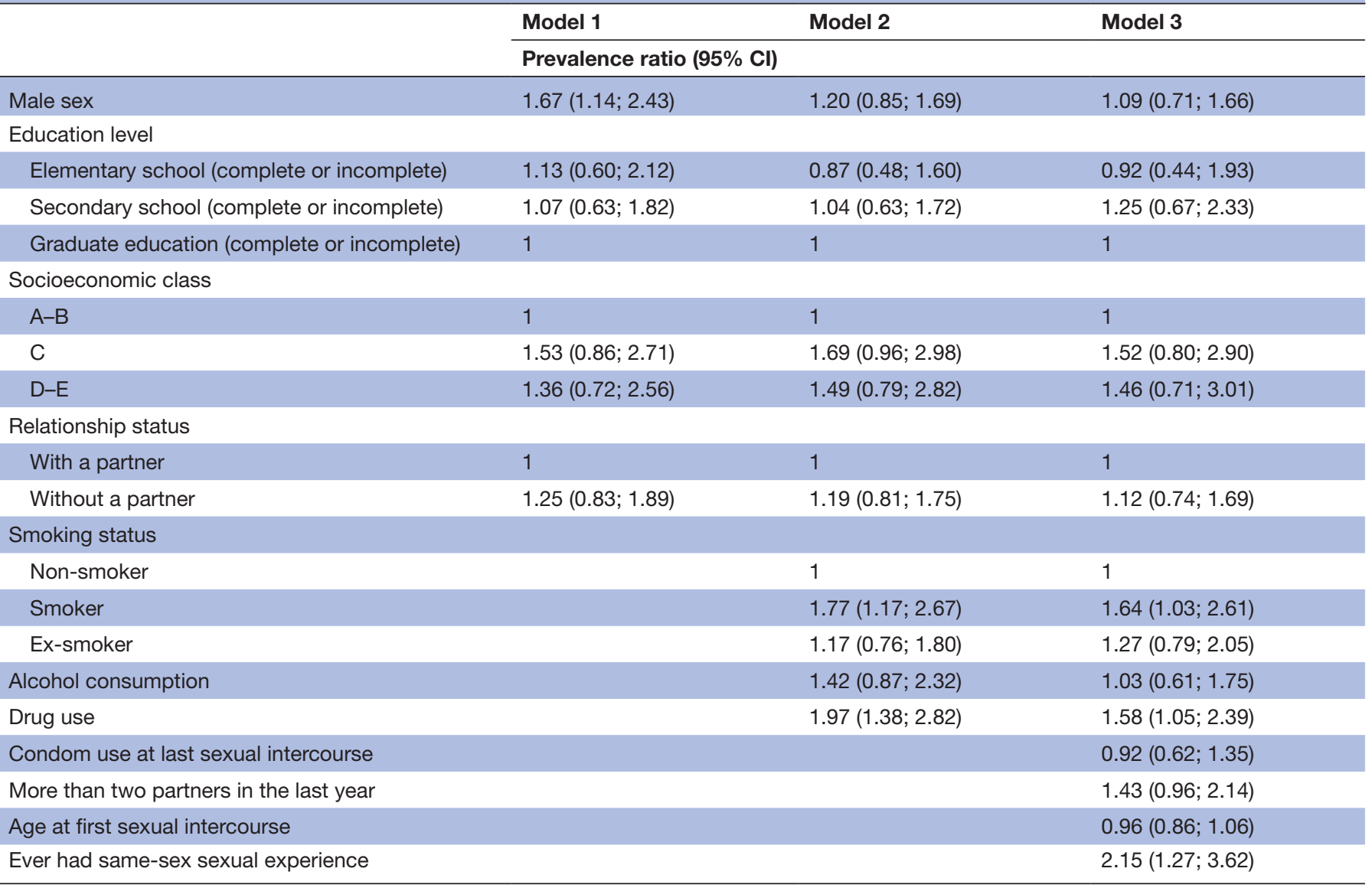

Model 1: Sex, education level, socioeconomic class and relationship status.

Model 2: Model 1+smoking status, alcohol consumption and drug use.

Model 3: Model 2+condom use at last sexual intercourse, more than two partners in the last year, age at first sexual intercourse and ever had same-sex experience.

HPV, human papillomavirus; STI, sexually transmitted infection. 
It was evident in the present study that smoking, drug use and ever having a same-sex sexual experience were uniquely associated with the presence of HPV/STI coinfection. Smoking cigarettes contributes to cervical cancer and has already been associated with the prevalence, incidence and persistence of HPV. ${ }^{29}$ Few studies have examined the relationship between drug use and HPV infection, but cocaine use has been associated with an increased risk of detection of both prevalent and incident oncogenic HPV infection, suggesting that the response to HPV infection might be altered by cocaine. ${ }^{30}$ Condom use seems not to interfere with the prevalence of HPV in drug users. ${ }^{31}$ Furthermore, same-sex sexual experience as a risk factor can be explained by the extended heteronormality in the healthcare system (and at Brazilian society) and unequal access to health services. ${ }^{32}$ No significant differences were found in relation to sociodemographic characteristics, despite the fact that STIs disproportionately affect low-income and middle-income countries. ${ }^{33}$

Although the rates of more than one STI associated with HPV were relatively low in the present study, the presence of one STI is itself a risk factor for contracting another STI. ${ }^{14}$ This is probably attributable to the impairment of the immune response by STIs, such as the presence of common risk factors. Another study of South African women showed that male sexual partners' STI histories may be more important than women's lifetime number of sexual partners. ${ }^{15}$ This factor was not analysed in the present study, but it could be important for the developing medical and behavioural interventions that include STI screening and treatment programmes, information on condom use and HPV vaccination for both women and men to reduce the prevalence of HPV/STI coinfection. ${ }^{15}$

This is the first study to assess factors associated with HPV and other self-reported STI coinfections in men and women in Brazil. The sociodemographic characteristics of the study participants were similar to those of the Brazilian population. ${ }^{11}$ Nevertheless, some limitations should be noted. We use the term coinfection even for infections (syphilis, gonorrhoea or herpes) that could be diagnosed and treated in the past. The infections may have occurred in the past and thus may not reflect the actual STI status. The cases of STIs were self-reported (with the exception of HIV), and many STIs have no signs or symptoms, both of which may have contributed to an underestimation of STI prevalence. Previous study with the same sample age estimated that $7 \%$ of all sexually experienced young men rescinded sexually transmitted disease self-reports over time. ${ }^{34}$ We use Roche Linear Array HPV genotyping, and this test have a consistently high sensitivity and specificity (91.7\% to $94.3 \%$, respectively). ${ }^{35}$

The results identified a low prevalence of self-reported STIs, but in participants with at least one infection, the prevalence of HPV was high. Smoking, drug use and same-sex sexual experience were associated with HPV/STI coinfection among Brazilian young adults. These findings reinforce the importance of implementing strategies to prevent risky behaviours. This knowledge may contribute to improving prevention strategies and treatment.

\section{Author affiliations}

${ }^{1}$ Escritório de Projetos PROADI-SUS, Hospital Moinhos de Vento, Porto Alegre, Rio Grande do Sul, Brazil

${ }^{2}$ Programa de Pós Graduação Endocrinologia, Universidade Federal do Rio Grande do Sul, Porto Alegre, Rio Grande do Sul, Brazil

${ }^{3}$ National Immunization Program, Ministry of Health, Brasilia, Brazil

${ }^{4}$ Department of STIs, AIDS and Viral Hepatitis, Ministry of Health, Brasilia, Brazil

${ }^{5}$ Instituto do Câncer do Estado de São Paulo, Universidade de São Paulo and Cancer Institute of the São Paulo State (ICESP), São Paulo, Brazil

${ }^{6}$ Faculdade de Medicina, Universidade de São Paulo, São Paulo, São Paulo, Brazil ${ }^{7}$ Department of Community Health, Federal University of Health Science of Porto Alegre, Porto Alegre, Rio Grande do Sul, Brazil

Contributors NLK and EMW contributed substantially to the conception and design of the work. ASB, CD, AGKM and FMAS contributed to conception of the work. NLK wrote the first draft. MB conducted the data analyses. BM and LLV conducted the lab analyses. All authors contributed to the interpretation of the data and approved the final version published, with EMW, JDCH, GFMP and LLV primarily responsible for revising the drafts for publication.

Funding This work was supported by the Hospital Moinhos de Vento in a partnership with the Department of Surveillance, Prevention and Control of Sexually Transmitted Infections, HIV/AIDS and Viral Hepatitis, of the Secretariat for Health Surveillance of the Brazilian Ministry of Health through the Institutional Development Support Program of the Brazilian National Health System (PROADI-SUS).

Competing interests None declared.

Patient consent for publication Not required.

Provenance and peer review Not commissioned; externally peer reviewed.

Data sharing statement Extra data are available by emailing elianawend@gmail. com.

Open access This is an open access article distributed in accordance with the Creative Commons Attribution Non Commercial (CC BY-NC 4.0) license, which permits others to distribute, remix, adapt, build upon this work non-commercially, and license their derivative works on different terms, provided the original work is properly cited, appropriate credit is given, any changes made indicated, and the use is non-commercial. See: http://creativecommons.org/licenses/by-nc/4.0/.

\section{REFERENCES}

1. WHO. Global health sector strategy on Sexually Transmitted Infections, 2016-2021. World Health Organization Department of Reproductive Health and Research, 2016.

2. Dunne EF, Unger ER, Sternberg M, et al. Prevalence of HPV infection among females in the United States. JAMA 2007;297:813.

3. Ayres AR, Silva GA. Cervical HPV infection in Brazil: systematic review. Rev Saúde Públ 2010;44:963-74.

4. McQuillan G, Kruszon-Moran D, Markowitz LE, et al. TPrevalence of HPV in Adults Aged 18-69: United States, 2011-2014. NCHS Data Brief 2017:1-8.

5. INCA - Instituto Nacional de Câncer - Estimativa 2018 - Introdução. http://www.inca.gov.br/estimativa/2018/introducao.asp (Accessed 26 Apr 2018).

6. ICO/IARC Information Centre on HPV and Cancer (HPV Information Centre). Human Papillomavirus and Related Diseases in the World. Summary Report 2017;27.

7. Lin C, Franceschi S, Clifford GM. Human papillomavirus types from infection to cancer in the anus, according to sex and HIV status: a systematic review and meta-analysis. Lancet Infect Dis 2018;18:198-206.

8. Castellsagué X, Bosch FX, Muñoz N. Environmental co-factors in HPV carcinogenesis. Virus Res 2002;89:191-9.

9. Wendland EM, Caierão J, Domingues C, et al. POP-Brazil study protocol: a nationwide cross-sectional evaluation of the prevalence and genotype distribution of human papillomavirus (HPV) in Brazil. BMJ Open 2018;8:e021170.

10. Bastos FI. Barata R de CB, Aquino EM, et al. Comportamento sexual e percepções sobre HIV/Aids no Brasil. Rev Saúde Públ 2008;42:1-2. 
11. Instituto Brasileiro de Geografia e Estatística - IBGE. Censo Demográfico. 2010:1-251.

12. Global Adult Tobacco Survey Collaborative Group. Tobacco Questions for Surveys: A Subset of Key Questions from the Global Adult Tobacco Survey (GATS). 2 edn. Atlanta, GA: Centers for Disease Coltro and Prevention, 2011.

13. Associação Brasileira de Empresas de Pesquisa. Critério de classificação econômica Brasil 2015 http://www.abep.org/criteriobrasil (accessed 27 Feb 2018).

14. Mbulawa ZZA, van Schalkwyk C, Hu NC, N-c H, et al. High human papillomavirus (HPV) prevalence in South African adolescents and young women encourages expanded HPV vaccination campaigns. PLoS One 2018;13:e0190166.

15. Menezes LJ, Pokharel U, Sudenga SL, et al. Patterns of prevalent HPV and STI co-infections and associated factors among HIVnegative young Western Cape, South African women: the EVRI trial. Sex Transm Infect 2018;94:55-61.

16. Amorim AT, Marques LM, Campos GB, et al. Co-infection of sexually transmitted pathogens and Human Papillomavirus in cervical samples of women of Brazil. BMC Infect Dis 2017;17:769.

17. Comar M, Monasta L, Seraceni S, et al. Chlamydia trachomatis and HPV co-infections in HIV negative men from a multi-ethnic area of Northern Italy at high prevalence of cervical malignancies. $J$ Med Virol 2017:89:1654-61.

18. Qian HZ, Hu Y, Carlucci JG, et al. Human Immunodeficiency Virus Status Differentially Associated With Genital and Anal Human Papillomavirus Infection Among Chinese Men Who Have Sex With Men: A Cross-Sectional Survey. Sex Transm Dis 2017;44:656-62.

19. Bosch FX, Lorincz A, Muñoz N, et al. The causal relation between human papillomavirus and cervical cancer. J Clin Pathol 2002:55:244-65.

20. Koutsky LA, Holmes KK, Critchlow CW, et al. A cohort study of the risk of cervical intraepithelial neoplasia grade 2 or 3 in relation to papillomavirus infection. N Engl J Med 1992;327:1272-8.

21. Brickman C, Palefsky JM. Human papillomavirus in the HIV-infected host: epidemiology and pathogenesis in the antiretroviral era. Curr HIVIAIDS Rep 2015;12:6-15.

22. Ortiz AP, Tamayo V, Scorsone A, et al. Prevalence and correlates of cervical HPV infection in a clinic-based sample of HIV-positive Hispanic women. Papillomavirus Res 2017;4:39-44.
23. Tartaglia E, Falasca K, Vecchiet J, et al. Prevalence of HPV infection among HIV-positive and HIV-negative women in Central/Eastern Italy: Strategies of prevention. Oncol Lett 2017;14.

24. Araújo AC, Carvalho NO, Teixeira NC, et al. Incidence of cervical intraepithelial neoplasia in a cohort of HIV-infected women. Int J Gynaecol Obstet 2012;117:211-6.

25. Badial RM, Dias MC, Stuqui B, et al. Detection and genotyping of human papillomavirus (HPV) in HIV-infected women and its relationship with HPV/HIV co-infection. Medicine 2018;97:e9545.

26. Veldhuijzen NJ, Braunstein SL, Vyankandondera J, et al. The epidemiology of human papillomavirus infection in HIV-positive and HIV-negative high-risk women in Kigali, Rwanda. BMC Infect Dis 2011;11:333.

27. Burchell AN, Winer RL, de Sanjosé S, et al. Chapter 6: Epidemiology and transmission dynamics of genital HPV infection. Vaccine 2006;24 Suppl 3:S52-S61.

28. Smith JS, Herrero R, Bosetti C, et al. Herpes simplex virus-2 as a human papillomavirus cofactor in the etiology of invasive cervical cancer. J Natl Cancer Inst 2002;94:1604-13.

29. Schabath MB, Villa LL, Lazcano-Ponce E, et al. Smoking and human papillomavirus (HPV) infection in the HPV in Men (HIM) study. Cancer Epidemiol Biomarkers Prev 2012;21:102-10.

30. Minkoff H, Zhong Y, Strickler HD, et al. The relationship between cocaine use and human papillomavirus infections in HIV-seropositive and HIV-seronegative women. Infect Dis Obstet Gynecol 2008;2008:1-7.

31. Dev D, Lo Y, Ho GY, et al. Incidence of and risk factors for genital human papillomavirus infection in women drug users. J Acquir Immune Defic Syndr 2006;41:527-9.

32. Logie $\mathrm{CH}$, Lacombe-Duncan A, Wang $\mathrm{Y}$, et al. Sexual orientation differences in health and wellbeing among women living with hiv in canada: Findings from a national cohort study. AIDS Behav 2018;22:1987-2001.

33. Unemo M, Bradshaw CS, Hocking JS, et al. Sexually transmitted infections: challenges ahead. Lancet Infect Dis 2017;17:e235-e279.

34. Dariotis JK, Pleck JH, Sonenstein FL, et al. What are the consequences of relying upon self-reports of sexually transmitted diseases? Lessons learned about recanting in a longitudinal study. $J$ Adolesc Health 2009;45:187-92.

35. Xu L, Oštrbenk A, Poljak M, et al. Assessment of the Roche Linear Array HPV Genotyping Test within the VALGENT framework. J Clin Virol 2018;98:37-42. 